\title{
Resultados preliminares de la investigación "Las emprendedoras y los emprendedores en Ciencia y Tecnología en Costa Rica: un contraste de realidades de género"
}

\author{
Preliminary results of the investigation "Women \\ and Men Entrepreneurs in Science and Technology \\ in Costa Rica: a contrast of gender realities"
}

Eugenia Ferreto-Gutiérrez'

Rytha Picado-Arroyo ${ }^{2}$

Fecha de recepción: 6 de agosto del 2013

Fecha de aprobación: 3 de setiembre del 2013

Ferreto-Gutiérrez, E; Picado-Arroyo, R.

Resultados preliminares de la investigación

"Las emprendedoras y los emprendedores en

Ciencia y Tecnología en Costa Rica: un contraste

de realidades de género". Tecnología en Marcha.

Número Especial. Pág 84-93.

I. Administradora de Empresas. Escuela de Administración de Empresas, Instituto Tecnológico de Costa Rica. Tel. 2550-9058. Correo electrónico: eferreto@itcr.ac.cr

2. Administradora de Empresas. Escuela de Administración de Empresas, Instituto Tecnológico de Costa Rica. Tel. 2550-9270. Correo electrónico: rpicado@itcr.ac.cr 


\section{Eugenia Ferreto Gutiérrez}

Es la última de seis hijos del matrimonio conformado por Rolando Ferreto Monge y Ana Lucía Gutiérrez Aguilar.

Nació en San José un 12 de noviembre de 1975, sin embargo es en Cartago donde siempre ha residido, siendo esta la provincia quien le otorgó la educación pública, desde el nivel primario hasta la Universitaria.

Es en 1999 cuando ingresó a estudiar al Instituto Tecnológico de Costa Rica, institución de la cual obtiene los grados de Bachiller, Licenciatura y el finalmente la Maestría, todos en Administración de Empresas.

Inició su vida laboral trabajando para la empresa Ingeniería Guzmán, como asistente de la Gerencia General, posteriormente se incorpora al Programa de Técnicos en Administración de Empresas hasta el año 2004. Es en el año 2005, cuando inicia a trabajar para el ClE-TEC, estando los dos primeros años a cargo de la coordinación del área de Mercadeo, para tomar cargo de la Coordinación en el año 2007 hasta la fecha.

Es en este año, cuando también incursiona en el campo de la docencia, brindando el curso de Desarrollo de Emprendedores y Teoría Administrativa, además y como profesora guía o lectora de proyectos de graduación. Desde enero de 2010 forma parte del cuerpo docente de la Maestría en Gestión de la Innovación Tecnológica (MAGIT) de la Universidad Nacional.

Junto con las investigadoras Grettel Castro y Ana María Fournier realizan una investigación para los años 2007 y 2009 denominada "Transferencia y Transformación de Desechos Industriales". Es en 2012 y 2013 cuando junto con la investigadora Rytha Picado, llevan a cabo la investigación "Las emprendedoras y los emprendedores en Ciencia y Tecnología en Costa Rica: un contraste de realidades de género", cuyos resultados preliminares se muestran en esta edición. 


\section{Rytha Picado Arroyo}

Nació un 02 de marzo de 1972 en el barrio Caballo Blanco de Cartago y a los cinco años se traslada a Tejar de El Guarco, lugar donde vive su niñez y juventud y también se encuentra su domicilio actual. Única hija de Mario Picado Hidalgo y de Aida Arroyo Rojas.

Estudió en la escuela Ricardo Jiménez y en el Colegio Sagrado Corazón de Jesús en Cartago, hasta tercer año. En 1988 empezó su educación técnica cuando se traslada al Colegio Vocacional de Artes y Oficios (COVAO) a estudiar Artes Gráficas y es en 1991 con su título de Técnico Medio cuando inicia su vida laboral.

Posteriormente continuó con sus estudios universitarios en la Universidad Latina de Costa Rica donde obtuvo su título de Bachillerato en Publicidad. Inició su vida profesional desde muy joven, laborando para varias empresas e instituciones. Desarrolló experiencia durante quince años en el área de Mercadeo en empresas financieras y de ventas al detalle. En el año 2005 se gradúa como Máster en Administración de Empresas con énfasis en Mercadeo del Tecnológico de Costa Rica. También trabajó como consultora de empresas, en temas de Estrategia e Investigación de Mercados. Experiencia en el campo docente y de capacitación de más de 10 años en instituciones públicas y privadas.

Ingresa a laborar a la Escuela de Administración de Empresas del Tecnológico a tiempo completo desde enero del 2008 y desde entonces se ha desempeñado como profesora y apoyando diferentes programas e iniciativas. Actualmente, trabaja como Coordinadora del Programa Institucional de Emprendedores y de los programas de extensión relacionados con el tema a través de Fundatec.

Es profesora de planta en cursos de Teoría Administrativa, Mercadeo, Desarrollo del Espíritu Emprendedor y Publicidad y Promoción a nivel Técnico, de Bachillerato y Maestría. Investigadora del Centro de Investigación en Administración, Economía y Gestión Tecnológica (CIADEG-TEC) en áreas de emprendimiento e innovación.

Forma parte de la red de apoyo a pymes liderada por el Ministerio de Economía, Industria y Comercio (MEIC) y formó parte de la comisión redactora de la política pública de emprendimiento: Costa Rica Emprende en el año 20l0. Es asesora permanente del Centro de Incubación de Empresas del TEC. Desde febrero del 2012 participa en el proyecto BUILD: "Impulsando una cultura emprendedora y los vínculos universidad-empresa para el desarrollo en América Central", auspiciado por la Unión Europea y en el cual participan 12 socios centroamericanos.

Cree en su trabajo como una vocación de vida y una bendición. Disfruta y agradece profundamente la oportunidad y la responsabilidad de formar personas pues se nutre con la energía que los estudiantes transmiten. Se siente comprometida con todos los proyectos de investigación y extensión que contribuyan y aporten mejoras a las personas y empresas. Se considera emprendedora y luchadora incansable pero sobre todo, se considera una soñadora. 


\section{Palabras clave}

Emprendimientos; género; habilidades; impacto familiar; actitudes; perfil; diferencias; contraste; ciencia y tecnología.

\section{Resumen}

El proyecto es una investigación que busca demostrar los contrastes existentes entre los emprendimientos femeninos y los masculinos en empresas enfocadas en ciencia y tecnología en Costa Rica. Al consultar fuentes secundarias y expertos en el tema y profundizar en este sector, se encontró una carencia de información documentada que permita guiar acciones pertinentes para los diferentes actores involucrados a nivel de gobierno, empresa privada y sector académico.

En 2010, el Ministerio de Economía, Industria y Comercio (MEIC) lanza dos políticas públicas, una de fomento a las pymes y otra a los emprendimientos, que establecen el tema de género como eje transversal. Además, el Instituto Tecnolológico de Costa Rica (ITCR) ha definido el apoyo a las pymes y el espíritu emprendedor como una política institucional y eje transversal de su quehacer académico (III Congreso Institucional), lo cual motiva a incursionar en esta línea de investigación. Partiendo de lo anterior, se planteó una propuesta de investigación orientada a sentar las bases que permitan hacer un contraste de género en los emprendedores y sus iniciativas en ciencia y tecnología y determinar si éste muestra diferencias o similitudes que impacten el desempeño de sus empresas.

En este artículo, el lector encontrará los resultados obtenidos de los dos primeros objetivos planteados; en entregas posteriores se profundizará en los demás resultados de la investigación. Para recabar la información se utilizó una metodología cualitativa de casos, con el fin de poder contrastar las vivencias empresariales entre ambos géneros en Costa Rica.

\section{Key words}

Entrepreneurship; gender; abilities; family impact; attitudes; profile; difference; contrast; science and technology.

\section{Abstract}

The project is an investigation that seeks to demonstrate the contrasts of gender entrepreneurship on companies focused in Science and Technology in Costa Rica. By consulting secondary sources and experts on the topic, and deepen the field of Science and Technology. We have found a lack of documented information to take appropriate actions to guide the various actors involved at the level of Government, private sector and academia.

In 2010, the Ministry of Economy, Industry and Commerce (MEIC) launches two public policy of promoting SMEs and other enterprises to which state the issue of gender as a crosscutting issue.

In addition, the Institute Tecnolológico Costa Rica (ITCR) has defined support for SMEs and entrepreneurship as an institutional policy and transverse axis of its academic work (Institutional III Congress), which motivates venture into this line of research.

Based on the above, this research proposal pretend at laying the foundations to make a gender contrast in entrepreneurs and their initiatives in science and technology and determine whether the differences or similarities impact on the performance of their companies. 


\section{Introducción}

El emprendimiento es un tema ampliamente discutido y desarrollado en Costa Rica a través de los últimos años por distintas instituciones y organizaciones que participan en el ecosistema emprendedor nacional. En este contexto, el interés de las investigadoras al llevar a cabo este proyecto fue comprender la dinámica de creación y crecimiento de las empresas y los emprendedores que las crean y determinar si existen o no y cuáles son sus diferencias desde el punto de vista de género.

Para ello se utilizó una metodología cualitativa de casos de emprendedores masculinos y femeninos, con el fin de obtener información que resulte de utilidad para la toma de decisiones de las instituciones gubernamentales, privadas y académicas relacionadas con la ciencia y la tecnología en el país.

Se analizaron aspectos tales como: perfil del emprendedor y la emprendedora cuando se creó la empresa así como en la época actual, el impacto familiar sobre las actividades empresariales, las habilidades predominantes y las actitudes de los emprendedores para lograr establecer, si existe, una diferencia entre los emprendedores y el desempeño empresarial debido al género de las personas que lideran estas empresas.

En este artículo se presentan los resultados preliminares obtenidos en cuanto a la definición de las habilidades emprendedoras de quienes dirigen las empresas en ciencia y tecnología y la identificación de las actitudes de los emprendedores al inicio de la empresa y en la etapa actual, al cabo de los años.

Lo anterior pretende mostrar una aproximación inicial a los temas descritos anteriormente, por lo cual es importante indicar que presenta conclusiones preliminares que podrían ser complementadas una vez terminado el análisis total de la investigación.

\section{Metodología}

En función de los objetivos propuestos, al ser un estudio exploratorio y debido a la carencia de información sobre las emprendedoras en este sector, se decidió utilizar una metodología cualitativa de casos (Eisenhardt, 1989).

Se aplicaron 10 estudios de casos para mujeres y I0 para hombres que posean empresas en el área de ciencia y tecnología. Se aplicaron criterios de selección como: empresas lideradas y/o creadas por hombres y/o mujeres, ubicadas en la Gran Área Metropolitana (GAM), que se desarrollen en el área de la ciencia y la tecnología, con un número similar de años de creadas y tamaño, entre otros, con el fin de realizar un contraste efectivo.

Los resultados obtenidos por el estudio de casos plasman la realidad vivida por las empresas dirigidas por hombres y mujeres, así como las habilidades administrativas de unos y otras, los obstáculos que se presentaron desde el inicio de la empresa hasta el crecimiento y mantenimiento.

La recolección de la información se hizo por medio de entrevistas a profundidad, durante las cuales se visitó la empresa del emprendedor para conocer la realidad empresarial y poder obtener respuesta a las interrogantes planteadas por las investigadoras.

Finalmente, se elaboró un instrumento de recolección de información de acuerdo con la teoría analizada, abordando aspectos tales como habilidades, actitudes y perfil empresarial, entre otros. El expediente contempló aspectos como: nombre de la empresa, emprendedor a cargo, dirección, teléfono, documentación obtenida por la empresa, entre otros.

\section{Resultados obtenidos}

Se presentan los dos primeros objetivos de la investigación como datos preliminares en los cuales se empieza a esbozar una imagen de la situación vivida por las emprendedoras y los emprendedores en ciencia y tecnología en el país. Para una lectura y comprensión práctica y sencilla de los resultados, cada objetivo se presenta desde el punto de vista femenino seguido del masculino.

\section{Definición de habilidades emprendedoras \\ de quienes dirigen las empresas \\ en ciencia y tecnología}

\section{De las emprendedoras}

Las emprendedoras analizadas identifican principalmente cinco habilidades cuando iniciaron la empresa: su habilidad para buscar información, crear proyectos empresariales, identificar las necesidades 
del entorno, capacidad para conseguir recursos y su liderazgo.

Si se les compara con la situación actual, solamente se mantienen dos habilidades en forma constante durante el desarrollo de su carrera empresarial, las cuales se refieren a su capacidad para conseguir recursos y su liderazgo. Cabe destacar que más bien las empresarias consideran haber adquirido otras habilidades emprendedoras durante su trayectoria, referidas a: la dirección y gestión de la empresa, el desarrollo de redes de contacto y el conocimiento técnico del negocio.

Las entrevistadas comentan que ejercen un liderazgo fuerte motivadas por el compromiso y la pasión que sienten por su empresa pero con estilo participativo, pues evidencian buena comunicación y equilibrio al tomar decisiones en conjunto con sus socios y colaboradores.

Con respecto al uso de redes de contactos, las empresarias manifiestan que son muy importantes para el desarrollo y crecimiento del negocio, específicamente los contactos empresariales que fortalecen las relaciones y la participación en cámaras y asociaciones relacionadas con su actividad.

\section{De los emprendedores}

Al inquirir sobre las habilidades y competencias emprendedoras que se tenían al inicio de su actividad empresarial, los entrevistados presentan una inclinación por la búsqueda de información, redes de contacto, establecimiento de metas, creación de proyectos empresariales e identificación de las necesidades del entorno.

Al hacer la comparación con las habilidades y competencias que actualmente posee el emprendedor, y en base a los años de experiencia y al mando de la empresa, las respuestas se focalizaron en conocimiento técnico del negocio, dirección y gestión de la empresa y liderazgo.

Finalmente, se hizo hincapié en cuestionar sobre el tipo de liderazgo que ejercen en las empresas y cómo este es percibido por los colaboradores. De ello se extraen manifestaciones tales como: "el liderazgo en la empresa es poder motivar a la gente, y transmitir la misión que uno tiene del negocio y de cómo quiere que sea la empresa". Algunos entrevistados coinciden en que se debe ejercer un "liderazgo participativo y de puertas abiertas, donde los emplea- dos sientan la confianza de conversar con la gerencia"; además, se trata de "un tema de consensuar aunque se presiona un poco a los trabajadores a la hora de cumplir con los tiempos establecidos". Sin embargo, es importante resaltar que para uno de los entrevistados el liderazgo se debe ejercer con un "estilo autoritario siempre", lo cual ha sido eficaz en su caso y no está considerando cambiarlo.

La habilidad identificada como redes de contacto es la única opción que el conjunto de varones mencionó tanto al inicio de las actividades empresariales como en la actualidad.

\section{Identificación de las actitudes de \\ los emprendedores al inicio de la empresa y en la etapa actual}

En este apartado se analizaron varios ítems: actitudes personales, actitudes emprendedoras, actitudes hacia la gestión empresarial y valores. En términos generales, todos juntos representan las actitudes emprendedoras, no obstante, se considera importante mostrar los resultados en forma separada con el fin de profundizar en la información obtenida.

Al analizar las actitudes emprendedoras, se deben mencionar los grandes elementos que se consideraron para este subapartado, como son la toma de decisiones -si es individual o colectiva-, la socialización de las situaciones con otras personas y el miedo al fracaso.

\section{Subapartado:Actitudes personales}

\section{De las emprendedoras}

En cuanto a las actitudes personales, las mujeres empresarias se identificaron con las que consideraron como las tres más importantes, tanto al inicio como en la etapa actual de sus empresas. Las tres actitudes encontradas al inicio fueron: la motivación personal, la toma de riesgo y la iniciativa personal. En cuanto a las actitudes en la etapa actual, se mantiene únicamente la toma de riesgo como una actitud necesaria e inherente al proceso emprendedor, pues la toma de decisiones empresariales en un entorno cambiante involucra asumir riesgos en forma constante.

Es importante rescatar que las empresarias consideran haber adquirido otras dos actitudes personales muy relacionadas con el mejoramiento de su gestión como líderes empresariales: la capacidad de decisión y la estabilidad emocional. 
Una de las actitudes que se profundizó en las entrevistas fue justamente la toma de decisiones, tratando de identificar si las mujeres emprendedoras toman estas decisiones en forma individual o colectiva (consultando a la familia y/o socios) y la mayoría recalcó que toman las decisiones en conjunto, con gerentes, socios y/o familia.

\section{De los emprendedores}

Al hablar de actitudes personales, las opciones que más mencionaron los empresarios para cuando iniciaban su empresa fueron la iniciativa personal, la toma de riesgo y la motivación personal.

Cuando se trata de tomar riesgos, el emprendedor indica que analiza el entorno, discute sus ideas con los socios -si los hay- y finalmente determinan cual es la decisión idónea para la empresa, siendo en algunos casos de un riesgo moderado y en otros alto, lo cual va aunado a la motivación personal e individual, pasando por la motivación que representan los hijos y los padres hasta los colaboradores que día a día se esfuerzan por ofrecer sus conocimientos en pro del beneficio colectivo.

Por otra parte, al calificar las actitudes en la época actual, la única diferencia que se presenta es con la capacidad de decisión, permaneciendo la toma de riesgo y la motivación personal. Para esta actitud, los emprendedores coinciden en que los conocimientos adquiridos y la experiencia acumulada al estar al frente de su empresa les hacen tomar decisiones acertadas, cuyos beneficios impactan positivamente en su desarrollo, ya sea que se exprese como incremento en las ventas, posicionamiento en el mercado o punto de referencia empresarial.

\section{Subapartado: Actitudes emprendedoras}

Por su parte, el Global Entrepreneurship Monitor (GEM, 20I0) establece el Modelo del GEM, el cual identifica tres componentes principales del emprendimiento: actitudes emprendedoras, actividades emprendedoras y aspiraciones emprendedoras (Acs y Szerb, 2009). Una combinación óptima de estos componentes y su interrelación pueden conformar un sistema emprendedor que ayude al desarrollo económico.

El GEM establece además que las actitudes emprendedoras son actitudes positivas hacia el emprendimiento, pueden incluir el nivel de riesgo que la persona esté dispuesta a soportar o la percepción que estas tienen de sus propias habilidades, conocimientos y experiencia en la creación de un negocio.

\section{De las emprendedoras}

Las actitudes emprendedoras mencionadas con mayor frecuencia por las empresarias al comienzo de su emprendimiento fueron: creatividad, visualización de oportunidades, generación de ideas e innovación.

Al analizar lo anterior a la luz de las actitudes emprendedoras mayormente mencionadas por las emprendedoras, se encuentra que coinciden con que dos de ellas continúan siendo las más importantes en su gestión empresarial: la visualización de oportunidades y la innovación. Los otros dos aspectos que se indican como los más importantes adquiridos por las emprendedoras a través del tiempo son la orientación hacia las metas y resultados específicos y el interés social.

Otro hallazgo importante es que al consultarle a las entrevistadas sobre cómo generan ideas o proyectos empresariales, la mayoría lo hace involucrando a los socios y el resto del personal. Además, también toman en cuenta a sus clientes pues consideran que las ideas provienen de las necesidades del mercado. La visualización de oportunidades de mercado básicamente surge de las necesidades de los clientes, por lo cual creen que deben estar constantemente revisando como se mueven sus sectores de interés.

La innovación la definen como hacer algo nuevo o mejorar algo ya existente, ya sea ideas, productos, servicios, procesos que surgen del mercado; por eso consideran que deben estar en constante búsqueda en su entorno y en el mercado para que sus empresas sean competitivas.

Las entrevistadas consideran que su mayor logro empresarial es haber desarrollado empresas que por su giro de negocio en ciencia y tecnología han logrado una posición en el mercado, han crecido y al día de hoy se mantienen en sus respectivas industrias.

\section{De los emprendedores}

Los emprendedores indicaron que las actitudes emprendedoras que poseían al inicio de su actividad 
empresarial eran: toma de riesgo, creatividad y orientación hacia metas y resultados específicos.

La actitud que más calificaciones obtuvo por parte del emprendedor es la toma de riesgo, el cual obtuvo expresiones como "es una muy buena lección de vida porque te ayuda, te enseña mucho y no los vuelves a cometer los problemas". Además, "se deben tomar riesgos, no hay que echarse para atrás, hay que seguir adelante y aunque no se encuentre toda la información disponible". Finalmente, otro emprendedor opinó que "miedo al fracaso no he tenido y hemos visto situaciones dificiles, no es que no tenga miedo al fracaso sino que simplemente considero que cualquier situación buena o mala siempre es una oportunidad".

La actitud emprendedora de la creatividad es una constante en varios de los emprendedores, ya que, como bien lo explican, el dinamismo en el que se ve inmerso el mundo empresarial actual no les permite permanecer inmóviles.

En la actualidad se muestra una variante en las actitudes seleccionadas por los emprendedores, pues se aprecia una inclinación hacia la innovación y el interés social.

Algunas de las respuestas ofrecidas por los emprendedores al cuestionarles sobre qué consideraban innovación son: "es lograr solventar problemas cotidianos mediante soluciones de perspectiva diferente, fáciles, sencillas de implementar y viables económicamente, es pensar en soluciones fuera de la caja, es el pensamiento divergente". Otro emprendedor la define como "estar cambiando, estar evolucionando todo el tiempo y máxime en el mercado en el que nos movemos nosotros, porque el que se duerme, en un mes, dos meses se quedó perdido, es ver oportunidades y aprovecharlas". Finalmente, un empresario la define como: "enfocarnos en la innovación y la innovación la interpretamos como una sustitución inteligente de importaciones de productos que se requieren en empresas de la Zona Franca que se formulan en Estados Unidos y que nosotros con nuestra experiencia y conocimientos podríamos desarrollarlos localmente".

La visualización de oportunidades, la tolerancia a la incertidumbre y la generación de ideas son las actitu- des emprendedoras que el sexo masculino indicó tener en ambas etapas empresariales.

Subapartado: Actitudes hacia

la gestión empresarial

\section{De las emprendedoras}

Resulta interesante analizar los resultados en lo que se refiere a las actitudes hacia la gestión empresarial, pues son exactamente las mismas al inicio y en la etapa actual de las empresas en ciencia y tecnología lideradas por mujeres. Estas actitudes son: el control, la independencia, el dominio sobre situaciones y el valor del tiempo.

Una de las actitudes hacia la gestión empresarial a la que se le prestó especial atención fue la aplicación de la delegación en sus empresas.

Otro de los aspectos consultados es cómo definen la priorización de las actividades de gestión empresarial. En este aspecto se encontró que las mujeres entrevistadas lo hacen a través del ordenamiento de tareas, roles y estructura organizacional para el logro de los objetivos. De esta manera, se evidencia gran claridad de gestión, sin importar el tamaño de la empresa y la cantidad de colaboradores.

Para concluir este apartado, se les consultó sobre el recurso financiero con el que inició la empresa. En este punto se observa que todas las empresas estudiadas iniciaron sus operaciones con recursos propios y en algunos casos con el apoyo de familiares o amigos cercanos. Esto concuerda con los resultados obtenidos por la II Encuesta Nacional desarrollada por el Observatorio de las Mipymes (20 I2), donde se plantea que el $80 \%$ de los emprendedores costarricenses iniciaron su empresa con fondos propios.

\section{De los emprendedores}

Tal y como sucedió en el caso de las mujeres, los empresarios manifestaron tener las mismas actitudes hacia la gestión empresarial tanto al inicio como en la etapa actual, ellas son independencia, control, dominio sobre situaciones y tiempo valioso.

Los emprendedores coinciden en que uno de los activos más valiosos que pueden tener al día de hoy es su independencia, que valoraron desde el primer 
día en que establecieron la empresa, ejerciendo su criterio y desarrollando las actividades según sus conocimientos.

La actitud de independencia en ambos tipos de emprendedores coincide con lo dicho por Varela (2008) sobre la existencia de una percepción de independencia y autodesarrollo que permita a las personas salir de sus posiciones de dependencia, lo cual requiere el desarrollo de los conceptos de autonomía y la valoración positiva de dicha independencia.

A la hora de preguntarles sobre la manera de efectuar la delegación, los empresarios manifiestan que es imposible no hacerlo. Para algunos fue un paso complicado pero lo lograron; únicamente tres emprendedores no lo hacen por las características particulares de las empresas.

\section{Subapartado:Valores}

\section{De las emprendedoras}

Las empresarias entrevistadas identificaron cuatro valores esenciales al inicio de la empresa: compromiso con el trabajo, constancia, perseverancia y responsabilidad personal; valores sin duda muy orientados al lanzamiento y desarrollo de los emprendimientos.

Al consultarles sobre los valores identificados en la etapa actual, la mayoría concuerda en que la perseverancia es el más importante de todos, seguido por el compromiso con el trabajo y la responsabilidad personal, todos ellos mencionados como valores esenciales al inicio de la empresa. Es decir, tres de los valores expresados al inicio se mantienen constantes a través del tiempo y la consolidación de las empresas.

No obstante, llama la atención que en la etapa actual se menciona la integridad como un valor más importante que la constancia, porque al crecer las empresas, se observa con mayor detalle el comportamiento ético con respecto a los clientes, los proveedores y la competencia.

\section{De los emprendedores}

Los emprendedores escogieron las opciones de honestidad, compromiso con el trabajo y perseverancia como los valores que poseían al inicio de su actividad empresarial. Los empresarios se autodenominaron como muy perseverantes, personas que no se rinden ante el primer obstáculo y continúan buscando opciones adecuadas para sus empresas.

En la actualidad, los emprendedores mantienen los tres valores antes mencionados y agregan a ellos la confianza y la constancia. De acuerdo con los entrevistados, ellos son los llamados a tener confianza en sus actividades y hacer que eso se refleje en su actuar, en el rumbo que lleva la empresa, esperando que impacte positivamente en los trabajadores, transmitiendo la constancia y la confianza que poseen sus líderes en los planes inmediatos y futuros.

\section{Conclusiones}

Las conclusiones sobre el primer objetivo, referido a las habilidades emprendedoras, son las siguientes:

Las mujeres muestran que han fortalecido sus habilidades emprendedoras, tales como las capacidades gerenciales, técnicas y de relaciones; factores sin duda fundamentales para garantizar la sostenibilidad de sus empresas.

Llama la atención que en todos los casos las emprendedoras no consideran relevante el uso de redes sociales como herramienta empresarial. En el caso de los hombres, consideran que las redes sociales no impactan, sin embargo, las redes de contacto personal, tales como reuniones, congresos, ferias y actividades, son una vitrina para el posicionamiento de la marca empresarial.

Con respecto al segundo objetivo, sobre las diferentes actitudes, se puede concluir que:

Al preguntar si las mujeres tienen temor al fracaso y cómo lo enfrentan, los resultados mostraron que las entrevistadas tienen una actitud decidida para enfrentarlo, seguridad en sí mismas y confianza en la experiencia generada por ellas y sus socios.

Las mujeres tienen una motivación para su mejora en la gestión empresarial, debido a su orientación hacia las metas. Con respecto a los resultados específicos y el interés social, se enfocan en el aporte que pueden hacer a la sociedad por medio de la creación de productos y servicios que satisfacen las necesidades de sus clientes.

Por su parte, las actitudes emprendedoras de los hombres se manifiestan en que cuatro de los diez casos estudiados tienen como insumo de genera- 
ción de ideas los aportes que puedan brindar los empleados. Las seis empresas restantes lo hacen desde la gerencia ejecutiva e informan a los subalternos sobre la manera en que se procederá.

En cuanto a la gestión empresarial por parte de las emprendedoras, se determinó que en casi todos los casos se practica la delegación, sobre todo con los colaboradores que tienen más tiempo de trabajar con la empresa, ya que son las personas con las que se ha desarrollado un mayor grado de confianza.

En este mismo subapartado, ambos géneros coinciden en que tienen una persona de confianza en quien depositan el accionar de la empresa y consideran que ejercerá un liderazgo positivo con los demás trabajadores.

\section{Bibliografía}

Acs y Szerb, (2009). Gearing Up to Measure Entrepreneurship in a Global Economy, Mimeo, Faculty of Business and Economics, University of Pecs.

GEM (20 I0). Reporte Nacional 20 I0: La situación del emprendimiento en Costa Rica.

Gómez, A. (2012). Informe Técnico de la segunda encuesta nacional de mipymes. San José: Observatorio de Micro, pequeñas y medianas empresas, UNED.

Lebendiker, M. (2010). Reporte Nacional 201 0: La situación del emprendimiento en Costa Rica. San José: Editorama.

Varela, R. (2008). Innovación Empresarial. Colombia: Pearson Prentice Hall. 